De l'espace à l'espèce dans le Manuscrit trouvé à Saragosse de Jean Potocki

\title{
Emilie Klene
}

\section{(2) OpenEdition}

\section{Journals}

Édition électronique

URL : http://journals.openedition.org/edl/467

DOI : 10.4000/edl.467

ISSN : 2296-5084

\section{Éditeur}

Université de Lausanne

\section{Édition imprimée}

Date de publication : 15 décembre 2012

Pagination : 183-194

ISBN : 978-2-940331-29-1

ISSN : 0014-2026

\section{Référence électronique}

Emilie Klene, « De l'espace à l'espèce dans le Manuscrit trouvé à Saragosse de Jean Potocki », Études de lettres [En ligne], 4 | 2012, mis en ligne le 15 décembre 2015, consulté le 18 décembre 2020. URL : http://journals.openedition.org/edl/467 ; DOI : https://doi.org/10.4000/edl.467 


\section{DE L'ESPACE À L'ESPÈCE DANS LE MANUSCRIT TROUVÉ À SARAGOSSE DE JEAN POTOCKI}

Cet article propose de mettre au jour le lien qui régit l'espace et le sujet dans le Manuscrit trouvé à Saragosse. Partant du postulat que, dans cette œuvre, le lieu semble constitutif de l'être, qu'il le conditionne jusque dans son identité la plus intime par l'influence des mœurs, de la filiation ou encore du climat, il semble intéressant d'interroger ce qu'est l'homme pour Potocki, indépendamment de son espace. Le lecteur est en effet le témoin privilégié d'une expérience sur l'humain menée à travers la Sierra Morena comme «laboratoire ontologique», dont il s'agit d'analyser les résultats.

Le héros du Manuscrit trouvé à Saragosse, Alphonse van Worden, doit se rendre de Cadix à Madrid pour y recevoir la commission aux gardes wallonnes. Il entend pour cela traverser la Sierra Morena, région espagnole fort inquiétante où «le voyageur qui se hasardait dans cette sauvage contrée [se] trouvait, disait-on, assailli par mille terreurs capables de glacer les plus hardis courages». Et en effet, la bravoure du jeune homme sera maintes fois mise à l'épreuve par la rencontre d'un homme possédé, de deux pendus, de succubes, ou encore par les récits effrayants des personnages qu'il y rencontre.

Alphonse van Worden: le seul nom du héros illustre par la mention du fief natal ("Worden») l'équation que la langue établit entre le sujet et l'espace dans lequel il évolue. Nombreux sont d'ailleurs les personnages du roman dont le nom - réel ou fictif - rappelle le lieu géographique: Trivulce de Ravenne, Apollonius de Thyane, Ménipe de Lycie, le duc d'Astorgas, Landulphe de Ferrare, etc. Que le patronyme porte la trace du lieu d'origine ne doit pas nous étonner. On sait notamment, grâce aux travaux d'Albert Dauzat, que les noms de famille sont en grande 
proportion des noms de provenance ${ }^{1}$. L'anthroponymie et la toponymie correspondent d'ailleurs aux deux parties de l'onomastique et elles entretiennent entre elles des liens étroits ${ }^{2}$. Ainsi le patronyme qui caractérise l'individu et ses descendants se fait par l'addition du nom du fief ou de la localité au nom de baptême, grâce aux prépositions "de", "du» ou "d'» ${ }^{3}$, particule distincte du nom chez les nobles et généralement soudée dans l'orthographe des noms roturiers. Par ailleurs, on parle bien de la racine des mots, rappelant par là même l'intrication du lieu et du langage. Le fait résulterait donc chez Potocki d'un procédé somme toute commun. Mais qu'il soit aussi fréquent dans le Manuscrit trouvé à Saragosse et qu'il s'applique même aux démons, aux empuses et autres créatures diaboliques telles la princesse de Monte-Salerno ou encore «la gente dariolette du châtel de Sombre», voilà de quoi surprendre davantage, comme si, semble-t-on nous dire, l'espace était le principal facteur identitaire de l'être.

\section{L'espace ou le conditionnement de l'être}

La mention géographique a en effet son importance. Certains personnages d'ailleurs changent d'état ou d'identité en changeant de lieu. La prudence peut l'imposer au cabaliste qui le précise en ces termes:

I. A. Dauzat, Les Noms de famille de France, p. I30. On appelle noms d'origine ou noms de provenance "ceux qui rattachent l'individu au sol, à la maison ou au domaine qu'il habite, à la localité ou au pays dont il est originaire».

2. M. Mulon, Origine et histoire des noms de famille, p. Io. Le lieu peut en effet désigner tout autant le lieu d'origine de l'individu surnommé, le lieu qu'il a quitté, celui où il habite ou encore celui auquel son activité le lie.

3. A. Dauzat, Les Noms de famille de France, p. 130 sq.: "C'est toutefois chez les nobles que l'usage d'ajouter le nom de la terre s'est d'abord développé et a pris de l'extension [...]. La "particule» de n'est nullement un signe de noblesse [...]. En revanche, de très nombreux roturiers ont des noms de modèle identique à ceux des nobles. Mais, en fait, l'immense majorité de l'aristocratie a pris la particule, tandis que l'usage s'est établi de bonne heure de souder $d e, d u$ ou $d$ 'dans l'orthographe des noms roturiers (Dubois, Deviller, Destrée). Aussi, la particule est-elle devenue signe de noblesse, à tel point que dès la monarchie des Bourbons, l'anoblissement la conférait et que, à partir du XVII ${ }^{\mathrm{e}}$ siècle tout au moins, les propriétaires roturiers prirent souvent l'habitude, pour se donner un air d'aristocrates, d'ajouter à leur nom celui d'une de leurs terres.» 
[...] ici je ne suis plus don Félix Uzeda, je suis Mamoun Ben Gerschon, juif de nation et de religion ${ }^{4}$.

Ou encore à Avadoro, ainsi que le rappelle la duchesse d'Avila:

[...] à Sorriente, vous avez été l'époux de Béatrice: à Madrid, vous êtes encore veuf de Léonore 5 .

Mais de manière plus fondamentale, les personnages du roman sont bien souvent conditionnés par cet espace dont ils portent en quelque sorte la trace, l'«impression» (terme par ailleurs leitmotiv dans le roman). Le cas d'Alphonse van Worden, né dans le fief éponyme, est à ce titre tout à fait représentatif. Le lieu de sa conception est le salon de compagnie transformé par les soins de son père, suite à une inondation domestique, en véritable camp fortifié:

Cependant son premier soin [= celui du père d'Alphonse] fut de placer à sec le lit de son épouse. Il y avait dans le salon de compagnie une cheminée à la flamande autour de laquelle quinze personnes pouvaient se chauffer à l'aise, et le manteau de la cheminée y formait comme un toit soutenu par deux colonnes de chaque côté. L'on boucha le tuyau de cette cheminée et sous son manteau, l'on put placer le lit de ma mère avec sa table de nuit et une chaise, et comme l'âtre était élevé d'un pied au-dessus, il formait une espèce d'île assez inabordable. Mon père s'établit de l'autre côté du salon sur deux tables jointes avec des planches et, de son lit à celui de ma mère, on pratiqua une jetée fortifiée dans le milieu par une espèce de batardeau, construit de coffres et de caisses. Cet ouvrage fut achevé le jour même de notre arrivée au château, et je suis venu au monde neuf mois après, jour pour jour ${ }^{6}$.

L'espace du salon, aménagé avec une rigueur digne d'un lieutenantcolonel aux gardes wallonnes, semble imprimer au jeune homme le sceau de l'honneur. Conçu dans un «assaut» aussi militaire qu'amoureux ${ }^{7}$, la nature du jeune Alphonse ne peut, semble-t-il, échapper au conditionnement de l'héroïsme. L'honneur, devenu une valeur quasi définitoire de

4. MTS-1810, p. 766.

5. MTS-1810, p. 750.

6. MTS-1804, p. 108.

7. Dans le droit fil de la rhétorique amoureuse qui, aux XVII et XVIII ${ }^{\mathrm{e}}$ siècles, exploite la richesse du langage guerrier. 
son être, dictera en effet toujours sa conduite, que ce soit pour emprunter l'itinéraire de la Sierra Morena ${ }^{8}$, ou encore pour réprimer sa crainte entre les deux pendus ${ }^{9}$. La fortification de fortune qu'est l'espace de sa genèse semble avoir aguerri le héros, protégé de la peur par le rempart de la bravoure, comme sa conception le fut par le rempart des caisses et des coffres.

Il est d'autres exemples plus significatifs encore. Le comte de Penna Velez souligne l'intrication toute particulière de l'espace et de la constitution du sujet. Le lien qui régit son lieu d'origine, la ville de Grenade, et la nature de son comportement, est très resserré. Voilà comment il introduit le récit de sa vie:

[...] vous savez que les poètes espagnols placent dans notre province le théâtre de toutes les scènes pastorales. Ils nous ont persuadés que notre climat devait inspirer l'amour et il n'est guère de Grenadin qui ne passe sa jeunesse et quelquefois sa vie entière sans autre occupation que d'aimer ${ }^{10}$.

Et en effet, lui qui se plaît à imaginer toutes sortes de tableaux de l'amour conjugal aussi idylliques que grotesques ${ }^{11}$, sera victime de sa sensibilité. Le climat grenadin, si favorable aux préoccupations amoureuses ${ }^{12}$, agit particulièrement sur lui, ainsi qu'il le confie à ses auditeurs:

8. MTS-1810, p. 60 sq.: «les lois de l'honneur me prescrivaient de me rendre à Madrid par le chemin le plus court, sans demander s'il était le plus dangereux».

9. MTS-1810, p. 87 : «Il me semblait que les lois de l'honneur m'obligeaient plus que jamais à passer par la Sierra Morena."

IO. MTS-1810, p. 308.

II. MTS-1810, p. 309 sq.: "Entrais-je dans une maison, bien loin de m'occuper de la conversation, je me plaisais à imaginer que la maison était à moi, et j'y logeais ma femme. Je meublais son salon des plus belles toiles des Indes, de nattes de la Chine et de tapis de Perse sur lesquels je voyais déjà l'empreinte de ses pas. Je croyais voir aussi les carreaux où elle s'asseyait de préférence. Sortait-elle pour prendre l'air, elle trouvait un balcon orné des plus belles fleurs avec une volière peuplée des oiseaux les plus rares. [...] A la promenade, c'était même folie: si j'avais un ruisseau à passer, j'entrais dans l'eau jusqu'à mi-jambe; ma femme passait sur les pierres, s'appuyant sur mon bras, et récompensait mes soins par un sourire céleste. Les enfants me ravissaient: je n'en rencontrais pas un, que je ne le mangeasse de caresses, et une femme nourrissant le sien me semblait le chef-d'œuvre de la création...".

I2. L'influence du climat sur les personnages sera développée dans E. Klene, «L'espace méditerranéen du Manuscrit trouvé à Saragosse, l'omphalos de l'homme?». 
[...] mon cœur avait plus qu'un autre ressenti la tendre influence de notre climat ${ }^{13}$.

Et le comte n'aura de cesse de souffrir les fâcheuses conséquences d'une âme sujette au doux abandon de la rêverie. A l'extrême inverse mais avec la même intensité, l'espace dans lequel évolue Zoto est également propice à forger son caractère. Le brigand, célèbre dans toute la Sierra Morena, fonde même les raisons de son acte dans l'influence de la Sicile. C'est en effet le climat de son pays natal qu'il rend responsable de sa soif de vengeance inextinguible vis-à-vis du principino:

Je ne pensais plus guère aux injures que j'en avais reçues, mais le nom du lieu me rendit toute ma rancune. Ceci ne doit pas vous surprendre: dans nos climats, les cœurs sont implacables. Si le principino eût été dans son château, je crois que je l'aurais mis à feu et à sang ${ }^{14}$.

Ainsi l'espace semble laisser une véritable empreinte sur le sujet. Au-delà d'une seule influence, il semble le constituer, le modeler jusqu'à le définir dans sa réalité la plus intime. L'être ainsi estampillé se forge un code de conduite guidé par l'honneur, par l'ambition amoureuse ou encore par la vengeance qui peut s'ériger en facteur structurel de sa personne. L'espace inscrit en lui des manières d'être et de penser, des principes qu'il ne soumet pas au crible critique et qui s'apparentent bien souvent à des vérités établies ou à des qualités fatalement inscrites en sa nature profonde qu'il subit avec résignation. Il n'est sans doute pas anodin de constater à quel point le juif errant dans la version de 1804, condamné à ne jamais s'inscrire dans un espace particulier, est sans doute l'être le moins guidé par des règles fixes. Ce personnage en effet n'a de cesse de relativiser toutes les croyances et de réduire les dogmes, notamment ceux des religions révélées, à de simples événements inscrits dans un contexte historique particulier. Il est celui qui, détaché de tout espace et par là même de tout conditionnement, permet de mettre en regard les différentes croyances. Sa marche permanente et son éternel mouvement instaurent une rupture avec toute transcendance, unique et immuable.

I3. MTS-1810, p. 309.

I4. MTS-1804, p. 163. 


\section{L'identité à l'épreuve de l'espace}

Dès lors, le Manuscrit trouvé à Saragosse s'apparente en bien des points à un vaste laboratoire dans lequel des personnages cobayes subissent l'expérience d'un déplacement, d'un transfert dans un espace autre. Il s'agit d'observer l'individu ou du moins ce qu'il en reste, "mis en solution» - pour emprunter un terme au lexique de la chimie - dans la Sierra Morena, et à l'épreuve de son attaque corrosive censée dissoudre le conditionnement de l'espace d'origine. La région espagnole et ce qu'elle suppose de récits polyphoniques, favorise en effet un large questionnement de la part des personnages sur leurs propres valeurs. Le long voyage d'Alphonse est l'occasion de découvrir des mœurs différentes et de réfléchir aux nombreux problèmes axiologiques soulevés. Il découvre d'autres repères, d'autres croyances, d'autres religions, tout ce qui relève d'un autre "espace», jusqu'à vivre des situations où la norme est l'exacte opposée de la sienne. Vertus cardinales de sa vie, la religion catholique et l'honneur ${ }^{15}$ vont être éprouvés par les belles musulmanes ainsi que par le principe d'honneur sicilien de Zoto dont la radicale différence à celui transmis par le fief Worden s'exprime dans un magnifique oxymore:

Testalunga, le plus honnête et vertueux bandit qu'il y ait eu en Sicile ${ }^{16}$.

Alphonse est d'ailleurs bien souvent ébranlé par la découverte de la relativité des valeurs:

[...] ce qu'il avait dit me donnait beaucoup à penser. Il n'avait cessé de vanter l'honneur, la délicatesse, l'exacte probité de gens à qui l'on aurait fait grâce de les pendre. L'abus de ces mots, dont il se servait avec tant de confiance, brouillait toutes mes idées ${ }^{17}$.

En inscrivant les personnages dans l'immense laboratoire de la Sierra Morena, le roman soumet les sujets à une épreuve d'ordre ontologique. Existe-t-il un être en tant qu'être, indépendant de son espace? Autrement dit: qu'est-ce que l'être si ses valeurs, ses repères et ses croyances - en

I5. MTS-1810, p. 121: «[...] il me semble que de renoncer à sa religion ou d'abandonner ses drapeaux sont deux choses également déshonorantes».

I6. MTS-1810, p. 157

I7. MTS-1810, p. 140. 
résumé ce qui pourrait définir le sujet - sont conditionnés par son espace d'origine, et donc relatifs? L'individu est-il condamné à n'être que le produit de facteurs particuliers, géographiques, accidentels, ou bien existe-til une nature humaine véritablement affranchie de tout trait contingent? Cette interrogation n'est pas sans conséquence notamment lorsqu'elle se pose à un siècle hanté par l'idée d'universalité. Qu'en est-il en effet à une époque qui place toute sa confiance dans la raison, le progrès et qui rêve d'un universel humain qui remplacerait l'universel divin? Dans sa critique des Droits de l'homme formulée dans les Considérations sur la France, Joseph de Maistre dénonce cet universel qu'il considère comme illusoire:

La Constitution de 1795, tout comme ses aînées, est faite pour l'homme. Or, il n'y a point d'homme dans le monde. J'ai vu, dans ma vie, des Français, des Italiens, des Russes, etc.; je sais même, grâces à Montesquieu, qu'on peut être Persan; mais quant à l'homme, je déclare ne l'avoir rencontré de ma vie; s'il existe, c'est bien à mon insu ${ }^{18}$.

Selon Pierre-Henry Tavoillot ${ }^{19}$, les Lumières ont tenté d'identifier une nature humaine et y auraient répondu de trois manières différentes: la position matérialiste défendue notamment par Hume et Helvétius, qui suppose que la nature de l'homme réside dans son corps; la position de l'humanisme abstrait qu'incarnent Rousseau et Kant et qui identifie l'essence de l'homme dans sa capacité à s'arracher aux besoins naturels et aux déterminations de son histoire; enfin la position romantique qui reconnaît la nature humaine dans son individualité, c'est-à-dire dans une liberté incarnée dans une nature concrète, située hic et nunc. A défaut de théorie, quels sont les résultats de l'expérience menée par Potocki sur la nature des hommes littéralement déracinés, coupés de leur espace, que celui-ci soit filiation, nation ou climat?

18. J. de Maistre, Considérations sur la France, p. 96.

19. P.-H. Tavoillot, Le Crépuscule des Lumières. 


\section{L'espace et le sujet: nouveaux rapports}

Le premier cas relevé est ce qu'un chimiste interpréterait comme relevant d'un "état stationnaire" ${ }^{20}$. Le lien qui régit le sujet et l'espace est si resserré que l'expérience du changement occasionne chez les personnages une force de résistance rendant l'expérience nulle. Certains êtres perdurent dans leur état, inchangés, immuables, quel que soit leur nouvel environnement. Nous pensons ici particulièrement au clan des Velasquez, à commencer par Don Carlos, courtisan de Louis XIV. Les conséquences de la permutation de son destin avec celui de son frère, le scientifique Henrique, occasionnée par la distraction de ce dernier qui se trompe de nom en signant sa lettre, soulignent le caractère imperméable de leurs univers respectifs. Don Carlos, déraciné, plongé fortuitement dans un tout autre espace, celui de l'application et de l'étude, persistera dans son étourderie et sa légèreté. Incapable d'évolution dans les nouvelles possibilités qui lui sont offertes, il occasionnera le malheur général. Ce caractère hermétique, cette résistance à l'altérité semblent héréditaires puisque le fils de Henrique, Velasquez, rend lui aussi l'expérience vaine. Plongé dans l'espace de la Sierra Morena, il restera tout entier tourné vers son intériorité, dans son univers de calculs, manquant même de mettre en échec la machination des Gomelez.

Le second cas concerne des êtres qui, implantés dans un espace autre, sont victimes de dissolution. Tout aussi incapables d'évolution que les précédents, ils ne réchappent pas à leur introduction dans un nouvel univers. La pression qui s'exerce sur eux leur est alors fatale. C'est le cas de don Phelipe d'Avadoro ${ }^{21}$, le père du chef des Bohémiens. La régularité de chacune de ses actions et son mutisme total en font un automate incapable de survivre ailleurs que dans la solitude et l'ordre implacable

20. "Etat d'un système ouvert dans lequel les grandeurs physiques en chaque point du système n'évoluent pas en dépit d'échanges de matière ou d'énergie avec l'extérieur", FranceTerme, www.franceterme.culture.fr/FranceTerme/ibdex.htlm.

2I. MTS-1804, p. 227 sq.: «Mon père s'appelait don Phelipe d'Avadoro et il passait pour l'homme le plus grave et le plus méthodique de son temps. Il l'était même si fort que si je vous contais l'histoire de l'une de ses journées, vous sauriez aussitôt celle de sa vie entière ou du moins, de tout le temps qui s'est écoulé entre ses deux mariages. Le premier à qui je dois le jour, et le second qui causa sa mort par l'irrégularité qu'il mit dans sa manière de vivre." 
de son appartement de la rue de Tolède. C'est en ces termes que son fils analyse sa mort:

Ainsi finit un homme qui n'était point né avec ce degré de forces physiques et morales qui eût pu lui donner même une énergie commune. Une sorte d'instinct lui avait fait choisir un genre de vie proportionné à ses moyens. On le fit périr en voulant le jeter dans la vie active ${ }^{22}$.

Ces deux premiers résultats se révèlent nuls pour l'étude menée sur la nature humaine au sens où les personnages ne peuvent être analysés sans le conditionnement de leur univers d'origine. Dans l'impossibilité de se débarrasser de leurs déterminations, incapables d'évoluer dans l'espace expérimental qui leur est ouvert, ils précipitent la faillite d'une mise à nu de leur être et de leur condition.

Le dernier cas en revanche, de loin le plus intéressant, est ce que l'on pourrait interpréter comme un "effet isotopique» ${ }^{23}$. Il concerne les personnages suffisamment souples dans un espace étranger, perméables, «miscibles» pourrait-on dire, pour entraîner une réaction à interpréter. En favorisant l'expérience de l'altérité, la Sierra Morena les débarrasse de l'influence de leur espace premier pour révéler certains traits, semble-t-il, universels. Nous songeons bien entendu ici au héros, Alphonse, inscrit au cœur du processus de transformation, mais aussi à Avadoro puisque nul autre mieux que lui ne parvient à changer d'identité aussi souvent que d'espace. Vice-reine du Mexique à Olmedo ${ }^{24}$, belle pénitente au collège des Théatins ${ }^{25}$, puis duc de Sidonia au cimetière de Burgos ${ }^{26}$, mendiant à Madrid ${ }^{27}$, ou encore chevalier de Calatrava en rentrant de Naples ${ }^{28}$, il devient à Vienne le marquis Castelli ${ }^{29}$. Qu'est-ce donc que la nature humaine, une fois libérée du conditionnement de l'espace?

22. MTS-1810, p. 570.

23. "Modification d'une propriété physique ou chimique d'une espèce chimique résultant d'un changement de sa composition isotopique", FranceTerme, www.franceterme.culture.fr/FranceTerme/index.html.

24. MTS-1804, p. 311.

25. MTS-1804, p. 431

26. MTS-1804, p. 445.

27. MTS-1804, p. 496.

28. MTS-1810, p. 589.

29. MTS-1810, p. 770. 
Il appert que l'expérience de la Sierra Morena favorise un nouveau positionnement de l'être. Les personnages se libèrent de leur espace d'origine que l'on pourrait qualifier de transcendantal puisque les valeurs, les repères, les croyances - tout ce qui relève du sujet - sont transmis par l'autorité des pères ou celle des Ecritures. Jusque-là amarrés à un repère fixe, ils rompent leurs attaches et prennent conscience de l'espace horizontal, illimité, qui s'ouvre à eux. Brisant les frontières de l'univers borné aux niveaux géographique, social, religieux, ils mesurent ce qu'est l'étendue, et les possibilités qu'elle offre. L'espace n'est plus ancré dans une verticalité immuable qui fixerait le genre humain ici-bas, mais bien dans l'étendue aux contours flous et aux vérités toujours à construire. Dès lors la nature humaine semble bien être l'espace créé entre deux points, soi et l'autre, ou plutôt cet espace réservé en soi à l'autre: Avadoro fera place à toutes les identités, Alphonse accueillera en son sein la religion musulmane, et tous les personnages - le lecteur y compris - seront dépositaires de la parole de l'autre, par l'écoute des récits. Sujet et espace, voilà bien un difficile rapport, surtout si le sujet advient à travers l'espace de l'autre. C'est sans doute d'ailleurs en cela que la région espagnole est effrayante:

[...] cette chaîne de monts sourcilleux qui séparent l'Andalousie d'avec la Manche n'était alors habitée que par des contrebandiers, des bandits et quelques Bohémiens qui passaient pour manger les voyageurs qu'ils avaient assassinés [...]. Le voyageur, qui se hasardait dans cette sauvage contrée s'y trouvait, disait-on, assailli par mille terreurs capables de glacer les plus hardis courages ${ }^{30}$.

"Disait-on", "passaient pour»: le narrateur ne prend pas en charge la description mais il préfère laisser s'élever d'autres voix ${ }^{31}$. En introduisant du jeu entre le sujet et le prédicat, ces modalisateurs rendent la description instable et plus terrifiante encore que par les attributs qu'on lui prête. L'ondoiement de la parole de l'autre résonne ici comme une menace confuse qui plane sans jamais réellement se manifester. Ainsi la région espagnole subit elle-même l'expérience d'autrui, redoutable certes mais constitutive du sujet.

Emilie KLene

Université de Montpellier, IRCL

30. MTS-1810, p. 59 sq.

3I. Voir à ce sujet l'article de D. Triaire, "Au commencement", à paraître. 


\section{BIBLIOGRAPHIE}

Textes

Maistre, Joseph (de), Considérations sur la France (1797), Paris, Imprimerie nationale Editions, 1994.

MTS-1804 et MTS-1810 = Ротоскі, Jean, Manuscrit trouvé à Saragosse (version de 1804 et version de 1810), éd. par François Rosset, Dominique Triaire, Paris, Flammarion, 2008 (GF 1342-1343).

\section{Travaux}

Dauzat, Albert, Les Noms de famille de France: traité d'Anthroponymie française, Paris, Guénégaud, 1977.

Klene, Emilie, "L'espace méditerranéen du Manuscrit trouvé à Saragosse, l'omphalos de l'homme?", in L'Invention littéraire de la Méditerranée au XIX ${ }^{e}$ siècle, Paris, Geuthner, à paraître.

Mulon, Marianne, Origine et histoire des noms de famille: essais d'anthroponymie, Paris, Errance, 2002.

Tavoillot, Pierre-Henri, Le Crépuscule des Lumières, Paris, Cerf, 1995.

Triaire, Dominique, "Au commencement", à paraître. 
